\title{
PRESERVACIÓN DE LA HERENCIA CULTURAL A TRAVÉS DEL TURISMO GASTRONÓMICO
}

\author{
CULTURAL HERITAGE PRESERVATION THROUGH \\ GASTRONOMIC TOURISM
}

\author{
María Cruz Cuevas Álvarez*, Marcos Pérez Mendoza**, \\ Enrique Pecero Covarrubias ${ }^{\star \star \star}$
}

\begin{abstract}
*Licenciada en Idiomas con terminal en turismo. Maestra en Docencia. Doctorante en Administración Educativa. Docente de la División Académica de Ciencias Económico Administrativas.

**Licenciado en Relaciones Comerciales. Maestro en Tecnología Educativa. Doctorante en Ciencias Administrativas. Docente de la División Académica de Ciencias Económico Administrativas.

***icenciado en Ingeniería Mecánico Electricista. Maestro en Administración de las Telecomunicaciones. Doctorante en Administración Educativa. Docente de la División Académica de Ciencias Básicas.
\end{abstract}

DIRECCIÓN PARA RECIBIR CORRESPONDENCIA: Correo electrónico: maria.cuevas@ujat.mx 
PRESERVACIÓN DE LA HERENCIA CULTURAL A TRAVÉS DEL TURISMO GASTRONÓMICO

RESUMEN

OBJETIVO: Resaltar la importancia del turismo gastronómico como alternativa de fuente de ingresos y como medio de brindar un valor agregado a un destino turístico.

MATERIAL Y MÉTODO: Para la realización del presente documento, se utilizó el método cualitativo con las técnicas: revisión documental de la literatura existente sobre el marketing gastronómico, tabla de contenido y observación participante.

RESULTADOS: Se ha identificado la diversidad de la cocina local e internacional como una de las ventajas competitivas de las ciudades o destinos turísticos; cada ciudad o destino aprovecha sus tradiciones culinarias, su cultura, sus festivales y misticismo con la finalidad de atraer turistas y brindar una experiencia única a paladares exigentes. Las personas que optaron por el Tour Teotihuacán, decidieron este servicio en búsqueda de una experiencia que combinara la cultura y la gastronomía del lugar, el cual está rodeado de historia y misticismo al considerarse este sitio arqueológico un lugar lleno de energía cósmica y haber sido una de las ciudades más importantes al albergar culturas florecientes, tales como: la teotihuacana y la mexica.

CONCLUSIONES: Siendo el arte culinario una actividad milenaria y rasgo distintivo de cada cultura, se ha convertido en la nueva herramienta mercadológica por servidores y proveedores turísticos, dándole importancia al conocimiento y valoración de las riquezas culturales, convirtiéndolo en el rasgo característico de cada destino turístico.

Palabras clave: Turismo gastronómico. Cultura. Destino turístico.

\section{ABSTRACT}

OBJECTIVE: To highlight the importance food tourism has an alternative income source and how to provide an added value to a tourist destination.

MATERIAL AND METHOD: The following qualitative article, was written down using the documentary revision on food marketing, content table and participant observation techniques.

RESULTS: Diversity on local and international food has been identified as one of the competitive advantages in cities and tourist destinations; each city or destination takes 
PRESERVACIÓN DE LA HERENCIA CULTURAL A TRAVÉS DEL TURISMO GASTRONÓMICO

advantage of its cooking traditions, culture, festivals and mysticism with the objective of attracting tourists and offer a unique experience to demanding palates. People, who chose The Teotihuacan Tour, selected this service in search of an experience that offered a combination of both culture and typical food, surrounded by history and mysticism, as this archaelogical site is considered to be full of cosmic energy and also being one the most important cities that held blossoming cultures, such as: teotihuacan and mexicas.

CONCLUSIONS: Being the culinary art a millennial activity and a distinctive feature of each culture, it has become the new marketing tool for tourist providers, giving importance to knowledge and giving value to cultural richness, making it the most important characteristic of each tourist destination.

Key words: Food tourism. Culture. Tourist destination.

\section{INTRODUCCIÓN}

En el mundo antiguo, la cocina de Mesopotamia era la más conocida, pero con el tiempo derivado de ella surgieron las cocinas de Anatolia y la de China. La cocina Anatolia es considerada la base de la cocina el antiguo Egipto, Grecia y Roma, siendo la cocina europea un derivado de la de Roma. En la actualidad, existen tres cocinas que son consideradas las mejores del mundo: la turca, la china y la francesa, por haber surgido a partir de un largo e histórico proceso (Aktas y Ozdemir, 2007, p. 23).

La gastronomía es un componente cultural importante, puesto que esconde en sus platillos años de tradición, recuerdos, momentos, personajes, técnicas y ambientes que hacen que cada lugar sea singular y especial (Valcálcer y Venegas, 2015, p. 2).

La gastronomía es vista como un campo de estudio científico por enfocarse en la relación existente entre la comida y la cultura. Por tener un rol crítico en el marketing de destino, por ser el factor de motivación determinante a considerar por los viajantes e incluso un elemento clave para reconsiderar el mismo destino nuevamente (Ozdemir y Seyitoglu, 2017).

Cuando se consume de la cocina extranjera, hay una implícita necesidad de aprender del contexto cultural en el cual la cocina se encuentra insertada (Biörk y Kauppinen-Räisänen, 2016; Henderson, 2009). Tal y como se ha evidenciado de manera conceptual y empírica en CUEVAS-ÁVAREZ M. C., PÉREZ-MENDOZA M., PECERO-COVARRUBIAS E. 
PRESERVACIÓN DE LA HERENCIA CULTURAL A TRAVÉS DEL TURISMO GASTRONÓMICO

trabajos previos, de que la mayor motivación por consumir la comida local de un destino turístico tiene que ver con la adquisición de conocimiento acerca de la historia a través de la experiencia auténtica y cultural que el destino brinda (Björk y Kauppinen-Räisänen, 2014; Fields, 2002; Kim y Eves, 2012; Kim et al., 2009).

Tanta atención ha atraído el turismo gastronómico que recientemente se ha convertido en el tema principal de la literatura académica, en especial sobre los factores y motivaciones en la toma de decisiones por parte de los turistas para la selección del destino turístico. Se ha demostrado que existen cuatro factores que motivan al consumo de la cocina local: fisiológicos, culturales, sociales y psicológicos (Fields, 2002; Kim y Eves, 2012; Kim et al., 2009; Mak, Lumbers, Eves y Chang, 2012).

Motivaciones que están influenciadas por variables que pueden hacer que los turistas cambien de idea con respecto a la degustación de la comida local: el género, la educación y la edad (Kim y Eves, 2012; Ryu y Han, 2010; Tse y Crotts, 2005); duración de la estancia, visitas repetidas (Tse y Crotts, 2005), así como conductas previas, exposición a la cultura local, normas subjetivas y actitudes (Ryu y Han, 2010).

Otros autores consideran que dichas motivaciones están relacionadas con el componente afectivo de la imagen y beneficios buscados (Dann, 1996; Gartner, 1993), el tipo de oferta (Figueredo, Ricon y Salazar, 2012), y variables sociodemográficas que pueden influir en el comportamiento de compra del destino (Um y Crompton, 1990).

El turismo es una actividad humana que depende de diversas motivaciones dependiendo del interés del individuo, en función de la experiencia acorde a la edad y al estatus del turista (Hsu, Cai y Wong, 2007). El marketing se ha diversificado tanto, que dentro de la literatura se encuentra desde el marketing de productos y servicios, el marketing de destino y un nuevo y creciente concepto, el marketing gastronómico.

Cuando el marketing de destino o destino turístico ya no satisface totalmente las expectativas y necesidades de los viajantes, se requiere considerar y buscar alternativas que permitan mantener atractivo a un sitio turístico. Para ello, es importante saber reconocer las actitudes y el comportamiento del turista para poder crear un perfil para captar su atención y saber que ofrecerle, que otros destinos no puedan garantizar. 
PRESERVACIÓN DE LA HERENCIA CULTURAL A TRAVÉS DEL TURISMO GASTRONÓMICO

Se debe girar la mirada hacia la importancia que la comida y el vino tienen para el visitante, cuando busca una experiencia diferente dentro del espectro de destinos turísticos existentes, de ahí la importancia y la relación que tienen el turismo y la gastronomía (López-Guzmán, Uribe, Pérez y Ríos, 2017).

Una mirada hacia el interior del destino, permite analizar y valorar la herencia cultural con la que se cuenta como lo son las costumbres y las tradiciones, que se ven reflejadas en una actividad diaria y tal vez sobrevalorada como lo es la cocina. En la cocina o la gastronomía de cada destino, se esconden riquezas culturales explotables. En cada una se cuenta su historia, sus creencias, sus recuerdos, sus personajes y sus valores; se denota un interés por aprender sobre el lugar que se visita, surge curiosidad por entender a la cultura a la que uno se inserta y todo esto se adquiere a través de esa experiencia auténtica y vivencial que el destino brinda a sus visitantes.

Tal y como lo hacen los prestadores de servicios de otras partes del globo, quiénes vieron a la cocina como un área de oportunidad con la finalidad de atraer y captar clientes reales y potenciales, un rasgo característico y único con el cual competir contra otros destinos.

El marketing gastronómico es entonces el siguiente paso a considerar con la finalidad de darle un valor agregado al destino, despertar la curiosidad del turista es responsabilidad de los involucrados en esta industria. Proveer servicios y productos auténticos y atractivos debe de ser la prioridad puesto que va en beneficio de la comunidad traduciéndose en un incremento de visitantes, derrama económica así como de visibilidad internacional del destino.

La planificación de un destino turístico debe hacerse en función de la oferta y la demanda, además de considerar también a las atracciones, los transportes, las instalaciones, los servicios, así como la promoción del turismo (Celis, Melián y Moreno Gil, 2002).

Una perspectiva a considerar, es el tipo de turistas que se desea atraer, por sobre la cantidad. Para lo cual, es imperativo, el que se identifique a los turistas reales de los potenciales, el que se determinen mercados meta, así como los productos y servicios adecuados para ofertar a cada segmento (Kotler, Gertner y Haider, 2007), al hacer esto, debe ser enfocado a la Teoría de Afinidad, la cual indica que hay un impacto en las intenciones de compra (Oberecker y Diamantopoulos (2011). 
PRESERVACIÓN DE LA HERENCIA CULTURAL A TRAVÉS DEL TURISMO GASTRONÓMICO

Cuando existen diversos destinos turísticos que basan su atractivo solamente en ciertos elementos, éstos pueden perder el interés de los visitantes con el tiempo por sus opciones limitadas (Butler, 1980), una alternativa para los que buscan el siguiente paso en la evolución del turismo, sería precisamente el turismo gastronómico (Kivela y Crotts, 2005).

La gastronomía es un elemento visible y tangible de una sociedad (Groce y Perri, 2010) se convierte en un elemento tanto auténtico como asequible (Reynolds, 1994), el cual contribuye al desarrollo y sostenibilidad del turismo (Scarpato, 2002). Fuertes beneficios económicos pueden generarse, si fuera la gastronomía la columna vertebral de la economía de una sociedad, al promoverse y venderse productos alimenticios (Belisle, 1983; Telfer y Wall, 1996; López y Martin, 2006) brindando con ello un valor agregado a cualquier destino turístico (Hall, Johnson y Mitchell, 2000; Hall y Sharples, 2003).

Tomando el ejemplo de Hillel, Belhassen y Shani (2013), al haber proveedores de vino en ruta en Negev, aprovechar esta autenticidad y ofrecer actividades de entretenimiento que involucren la producción de comida a través de la agricultura, en lugar de invitar a los paseantes a degustar, observar o a realizar otras actividades que pueden encontrar en otros destinos.

Como medio para conseguir ingresos, haciendo uso del marketing gastronómico y que tienen mayor alcance, son los festivales gastronómicos tal y como lo mencionan López-Guzmán et al (2017) en el caso de Guayaquil, cuyo evento de cuatro días generó 1.3 millones de dólares en su edición 2015, y con los que además se promueve un destino turístico para cierto tipo de turista.

Otro ejemplo de festival exitoso a nivel mundial es el Oktoberfest, realizado cada año desde 1810, entre los meses de septiembre y octubre, con duración de una semana en Alemania. Aunque su producto principal es la cerveza, actualmente hay toda una logística alrededor de este festival (Rockwood, 2011) y cuyo número de visitantes asciende a los 5.6 millones de visitantes, cifras obtenidas del Oktoberfest 2016 (Oktoberfest, 2017).

El creciente interés por la cocina local y la gastronomía, no sólo tienen un impacto en los ingresos económicos turísticos, sino también en el interés por conocer otra variedad de bebidas y alimentos de otros países, ya sea por motivos concernientes a la nutrición y a la salud, o simplemente porque la cocina o gastronomía está fuertemente relacionada con el estilo de vida de cada cultura (Guzel, 2015). Un gran número de turistas mencionan que el sabor de la comida local del lugar visitado, y es una parte importante de la experiencia de viaje ya que es CUEVAS-ÁVAREZ M. C., PÉREZ-MENDOZA M., PECERO-COVARRUBIAS E. 
PRESERVACIÓN DE LA HERENCIA CULTURAL A TRAVÉS DEL TURISMO GASTRONÓMICO

imposible conocer la cultura de la región sin degustar la cocina local (Yun, Hennessey y MacDonald, 2011).

\section{MATERIAL Y MÉTODO}

Para la realización de este trabajo se utilizó el método cualitativo utilizando la técnica de investigación documental (Rojas, 2011) sobre el marketing gastronómico, el análisis de contenido (Piñuel, 2002; Porta y Silva, 2003) y la observación participante (Martínez, 2009) durante un tour hacia Teotihuacán.

A partir de la revisión del estado del arte, se encuentra que se debe conocer la conducta y las motivaciones del turista, haciendo uso del modelo teórico de Yiannakis y Gibson (1992), una vez determinado el perfil llegar a la implementación de un apropiado plan de marketing gastronómico.

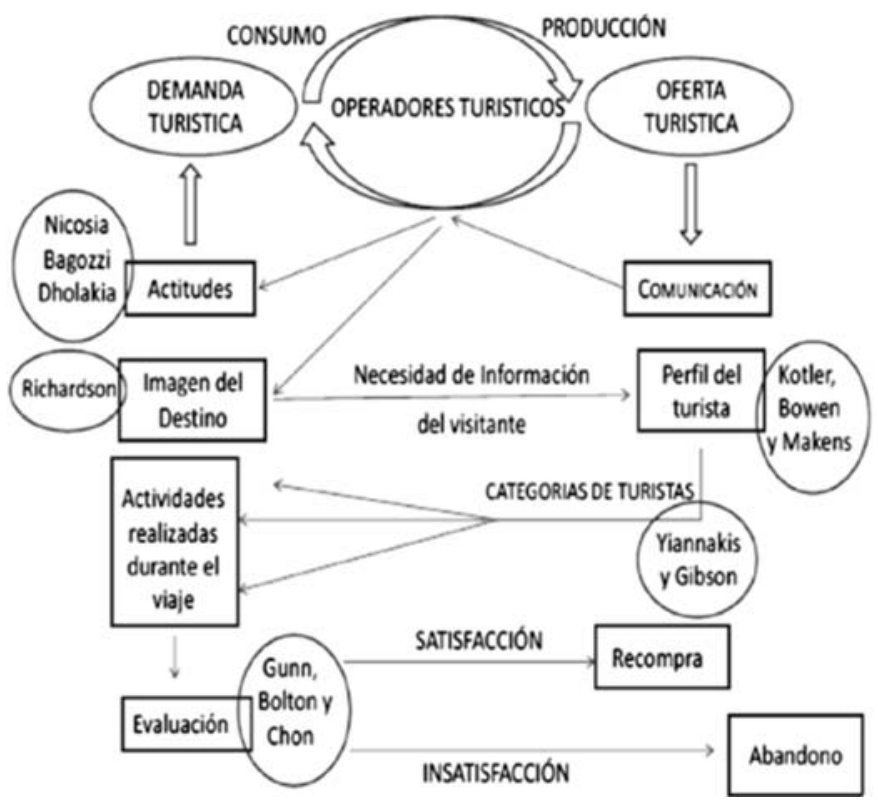

Figura 1. Modelo teórico de evaluación sistémica del comportamiento del consumidor turístico.

Fuente: Giraldo y Otero (2011). 
PRESERVACIÓN DE LA HERENCIA CULTURAL A TRAVÉS DEL TURISMO GASTRONÓMICO

\section{RESULTADOS}

La Organización de Marketing de Destino Local (OMD) ha identificado la diversidad de la cocina local e internacional como una de las principales ventajas competitivas de una gran ciudad como lo es Hong Kong. Con alrededor de 30,000 restaurantes, bares y outlets de comida, donde se ofrece todo tipo de comida china, asiática y occidental.

A unos kilómetros de Asia, en las Maldivas se aprovecha la mística que existe alrededor de su cultura. El país divulga orgullosamente sus ricas tradiciones culinarias mezclado con las bebidas, la cultura, las tradiciones y festivales permite la creación de paquetes dinámicos para los turistas brindándole valor agregado a la comunidad y a la industria turística del lugar (Hussain, 2012).

Por su parte, Hong Kong comparte sus números en cuestión de negocios de comida que brindan servicio tipo bares, restaurantes y outlets de comida, lo cual puede dar una idea de la cantidad de visitantes con los que cuentan.

En el caso de Turquía, la variedad de su cocina se relaciona con factores como la ubicación geográfica y procesos históricos lo que ha permitido el enriquecimiento y cambio de la misma. Con influencia árabe y asiática, la cocina turca está considerada como una de las 3 mejores y más importantes por su nivel nutritivo a nivel internacional (Guzel, 2015).

Alemania brinda el ejemplo perfecto de marketing gastronómico, además de incluir toda una logística alrededor de su festival y cuya cantidad de visitantes incrementa año con año (Rockwood, 2011). Guayaquil es otro destino gastronómico, cuyo festival es de reciente creación, reporta una creciente derrama económica.

Colombia transmite cultura en cada platillo que sirve, desde los tamales, el cuchuco, el sancocho, las empanadas, el chocolate santafereño, los dulce de arequipe, bocadillo y coco, se oculta su historia y su pasado. Entender como esos sabores han trascendido a lo largo del tiempo y descubrir cómo han logrado mantenerse por casi 197 años, y que chefs internacionales buscan realizar un sinnúmero de variaciones y mezclas de sus recetas comunes, para satisfacer paladares exigentes, hace que esta cultura y su cocina merezcan mención y reconocimiento (Valcálcer y Venegas, 2015). 
PRESERVACIÓN DE LA HERENCIA CULTURAL A TRAVÉS DEL TURISMO GASTRONÓMICO

En México, específicamente en la Ciudad de México, se encuentra una empresa turística, cuyos servicios además de incluir guía de turistas certificados, quiénes dominan un segundo idioma, ofrecen el servicio de tours hacia y durante la visita al sitio arqueológico de Teotihuacán, como valor agregado, durante el recorrido van indicando los sitios icónicos e históricos de la ciudad y brindan información o datos curiosos sobre ellos, similar al servicio que brindan los tours denominados turibuses.

La información que comparte en la zona arqueológica, evitan brindar información considerada clásica o esperada, como la que se puede obtener leyendo los señalizadores. Los guías brindan información actualizada, fundamentada y con referencias de artículos y libros de arqueólogos reconocidos e inclusive de quiénes se encuentran trabajando actualmente in situ, así como los hallazgos y la tecnología empleada en los nuevos descubrimientos del lugar. Adicional a esta información, el prestador del servicio o guía de turistas, durante el recorrido explica sobre la gastronomía que los nativos de lugar consumían, creando curiosidad entre los presentes e invitándolos a cuestionar sobre otros aspectos culturales de la civilización que habitaba el lugar.

Esta empresa mayormente cuenta con turistas extranjeros y los prestadores del servicio o guías de turistas denotan que el turista nacional no se preocupa por conocer sus raíces y menos por vivir este tipo de experiencias. También mencionaron durante el tour, que a pesar de que el turista nacional que vive en dicha ciudad, desconoce datos curiosos, históricos o relevantes de los lugares que ellos van indicando durante el recorrido y aprovecharon esta área de oportunidad para incluirla como un valor agregado a su servicio. Normalmente por tour, atienden entre 30 y 40 turistas extranjeros entre los que destacan: chilenos, peruanos, colombianos, franceses, alemanes e italianos, clientes que buscan experiencias donde la cultura y la gastronomía sean los atributos sobresalientes. Rara vez han brindado el servicio a turistas provenientes de Estados Unidos y México, siendo estos pocos de cierto segmento demográfico y económico: adultos mayores y jubilados.

Finalmente, después de la parte cultural, viene la parte gastronómica y se realizó una parada en un lugar donde producen tequila y mezcal. En este sitio, se les da una breve pero informativa plática sobre la planta de agave, así como los productos que se obtienen de ella, que son el tequila y el mezcal. Materiales con los que los ancestros creaban herramientas y diversos objetos para uso estético. 
PRESERVACIÓN DE LA HERENCIA CULTURAL A TRAVÉS DEL TURISMO GASTRONÓMICO

Como otro valor agregado, se brinda una degustación de diversos tipos de mezcal, para tomar en la forma acostumbrada por la cultura mexicana, haciendo ciertos movimientos corporales al mismo tiempo que se van diciendo frases típicas al beber dicho líquido; terminando el recorrido con una degustación de comida y bebida típica, momento en el cual convergen la cultura, la historia y la gastronomía en cada bocado y en cada sorbo de México.

\section{CONCLUSIONES}

La cocina, actividad milenaria y rasgo distintivo de cada cultura, es la nueva herramienta mercadológica utilizada por los servidores y proveedores de productos y servicios. Conocer y valorar las riquezas culturales con los que un destino cuenta, se traduce en la explotación de sus atributos que resultará en derrama económica del destino turístico, además de ofrecerlos como medio de conocimiento de años de historia y lograr tener visibilidad internacional.

Aunque no es una tarea fácil, no es una meta imposible de cumplir. Los destinos turísticos deberían considerar al marketing gastronómico como la excusa perfecta o la oportunidad de reinventarse, de atreverse a ofrecer lo que los demás no pueden por falta de atributos, limitaciones o de autenticidad.

El saber reconocer el perfil del visitante, así como sus deseos y motivaciones, son la pauta para refrescar los métodos mercadológicos empleados, tener perspectiva, acercarse al segmento de otra manera es la oportunidad perfecta para desarrollar el marketing adecuado y distinguirse del gran espectro de opciones existentes en el mercado; es entonces una oportunidad de innovar para dar un despertar hasta al paladar más exigente. 
PRESERVACIÓN DE LA HERENCIA CULTURAL A TRAVÉS DEL TURISMO GASTRONÓMICO

REFERENCIAS BIBLIOGRÁFICAS

Aktas, A. y Ozdemir, B. (2007). Otel işletmelerinde mutfağın Yönetimi. Ankara: Detay Yayıncılık.

Bélisle, F.J. (1983). Tourism and food production in the Caribbean. Annals of Tourism Research, 10(1), 497-513.

Björk, P., \& Kauppinen-Räisänen, H. (2014). Culinary-gastronomic tourism-a search for local food experiences. Nutrition \& Food Science, 44(4), 294-309.

Björk, P., \& Kauppinen-Räisänen, H. (2016). Exploring the multi-dimensionality of travellers' culinary-gastronomic experiences. Current Issues in Tourism, 19(12), 1260-1280.

Butler, R. W. (1980). The concept of the tourist area life cycle of evolution: implications for management of resources. Canadian Geographer, 24(1), 5-12.

Celis Sosa, D., A. Melián Gonzáles y S. Moreno Gil (2002). La planificación turística de un destino turístico: el caso de San Bartolomé de Tirajama. Las Palmas de Gran Canaria: Universidad de Las Palmas de Gran Canaria.

Dann, G. M. (1996). Tourist images of a destination - an alternative analysis. Journal of Travel \& Tourism Marketing, 5(1), 41 - 55.

Fields, K. (2002). Demand for the gastronomy tourism product: Motivational factors. In A. Hjalager, \& G. Richards (Eds.), Tourism and gastronomy (pp.37-50). London: Routledge.

Figueredo, C. A., Ricon, N. S., y Salazar, N. S. (2012). Inteligencia de mercados de productos Provincia de Tundama, Departamento de Boyaca, con Fines de Exportación. Ciencias Estratégicas, 20(27), 67-76.

Gartner, W. (1993). Image formation process. Journal of Travel and Tourism Marketing, 2(1), 191-212.

Groce, E. y Perri, G. (2010). Food and wine tourism: integrating food, travel and tourism. Oxfordshire: CABI.

Guzel, G. (2015). Gastronomy tourism as an alternative tourism: An assessment on the Gastronomy tourism potential of Turkey. International Journal of Academic Research in Business and Social Sciences, 5(9), 1-18.

Hall, C.M., Johnson, G. y Mitchell, R. (2000). Wine tourism and regional development en C.M. Hall, L. Sharples, B. Cambourne y N. Macionis (eds). Wine Tourism around the world: 
PRESERVACIÓN DE LA HERENCIA CULTURAL A TRAVÉS DEL TURISMO GASTRONÓMICO

Development, management and markets (pp. 196-225). Oxford: Butterworth-Heinemann.

Hall, C.M. y Sharples, L. (2003). The consumption of experiences or the experience of consumption? An introduction to the tourism of taste, en C.M. Hall, L. Sharples, R. Mitchell y Macionis y B. Cambourne (eds). Food tourism around the world: Development, management and market (pp. 1-24). Oxford: Butterworth-Heinemann.

Henderson, J. C. (2009). Food tourism reviewed. British Food Journal, 111(4), 317-326.

Hillel, D., Belhassen, Y. y Shani, A. (2013). What makes a gastronomic destination attractive? Evidence from the Israeli Negev. Tourism Management, 36(1), 200-209.

Hsu, C., Cai, L., y Wong, K. (2007). A model of senior tourism motivations. Anecdotes from Beijing and Shanghai. Tourism Management, 28(5), 1262 - 1273.

Kim, Y., Eves, A., y Scarles, C. (2009). Building a model of local food consumption on trips and holidays: A grounded theory approach. International Journal of Hospitality Management, 28(3), 423-431.

Kim, Y. G., y Eves, A. (2012). Construction and validation of a scale to measure tourist motivation to consume local food. Tourism Management, 33(6), 1458-1467.

Kivela, J. y Crotts, J. (2005). Gastronomy tourism: a meaningful market segment. Journal of Culinary Science and Technology, 4(2/3), 39-55.

Kotler, P., D. Gertner, I. y Haider, D. (2007). Marketing internacional de lugares y destinos: estrategias para la atracción de clientes y negocios en Latinoamérica. México, D.F.: Pearson.

López, X. Y Martin, B.G. (2006). Tourism and quality agro-food products: An opportunity for the Spanish countryside. Tijdschrift voor Economische en Sociale Geografie, 97(2), 166-177.

Mak, A. H., Lumbers, M., Eves, A., \& Chang, R. C. (2012). Factors influencing tourist food consumption. International Journal of Hospitality Management, 31(3), 928-936.

Martínez, M. (2009). Ciencia y arte en la metodología cualitativa. México: Trillas.

Oberecker, E. M., Riefler, P., y Diamantopoulos, A. (2008). The consumer affinity construct: conceptualization, qualitative investigation, and research agenda. Journal of International Marketing, 16(3), 23-56. 
PRESERVACIÓN DE LA HERENCIA CULTURAL A TRAVÉS DEL TURISMO GASTRONÓMICO

Ozdemir, B. y Seyitoglu, F. (2017). A conceptual study of gastronomical quests of tourists: authenticity, safety and comfort? Tourism Management Perspectives, 23(1), 1-7.

Piñuel, J. L. (2002). Epistemología, metodología y técnicas del análisis de contenido. Estudios de sociolingüística, 3(1), 1-42.

Porta, L. y Silva, M. (2003). La investigación cualitativa: el análisis de contenido en la investigación educativa. Recuperado de: http://www.uccor.edu.ar/paginas/REDUC/porta.pdf.

Reynolds, P. (1994). Culinary heritage in the face of tourism. Progress in tourism, Recreation and Hospitality Management, 6(1), 189-194.

Rojas, I. (2011). Elementos para el diseño de técnicas de investigación. Tiempo de educar, 12(24), 277-297.

Ryu, K., y Han, H. (2010). Predicting tourists' intention to try local cuisine using a modified theory of reasoned action: The case of New Orleans. Journal of Travel \& Tourism Marketing, 27(5), 491-506.

Scarpato, R. (2002). Gastronomy as a tourist product: The perspective of gastronomy studies in A. M. Hjalager and G. Richards (eds). Tourism and Gastronomy. London: Routledge.

Telfer, D.J. y Wall, G. (1996). Linkages between tourism and food production. Annals of Tourism Research, 23(3), 635-653.

Tse, P., y Crotts, J. C. (2005). Antecedents of novelty seeking: International visitors' propensity to experiment across Hong Kong's culinary traditions. Tourism Management, 26(6), 965968.

Um, S., y Crompton, J. (1990). Attitude determinants in tourism destination choice. Annals of Tourism Research, 17(1), 59-75.

Valcálcer, D. M. y Venegas, A. (2015). La comida típica dentro de la internacionalización de la oferta gastronómica en Bogotá. Turismo y Sociedad, 15(1), 187-198.

Yun, D., Hennessey, S. M. y MacDonald, R. (2011). Understanding culinary tourists: segmentations based on past culinary experiences and attitudes toward food-related behavior. International CHRIE Conference-Refereed Track. Massachusetts: University of Massachusetts.

Rockwood, K. (1 septiembre de 2011). Oktoberfest 2016. Fast Company. p. 26. Oktoberfest 2017. (2017) Recuperado de: http://www.oktoberfest.de/en/. 